barrier. One would thus achieve a substance which could be administered in an unobjectionable way (peripherally) and which would be expected to control the mental aberration.

After this work had been completed, a paper appeared ${ }^{11}$ describing effects of LSD25 on Siamese fighting fish. It was interesting to note that LSD-25 caused these animals to swim backward especially by action of the pectoral fins.

\title{
SUMMARY
}

The administration of lysergic acid diethylamide (LSD-25) to mice caused them to behave in a way similar to untreated mice faced with the prospect of sliding down an inclined plane. It was believed that the drug induced in the mice an hallucination of sliding down an inclined plane. This abnormal behavior was prevented in some of the animals by injection of serotonin plus carbamylcholine into the lateral ventricle of the brain. The carbamylcholine could be replaced by the cholinesterase inhibitor physostigmine. The serotonin plus carbamylcholine had to be given intracerebrally. Intraperitoneal injection was ineffectual. Even by the intracerebral route, however, it was not possible to counteract LSD-25 in all the animals. Detailed data showing the antagonism between serotonin and LSD-25 in isolated segments of carotid arteries were recorded. The behavioral change caused in mice by LSD-25 was viewed as resulting from a cerebral deficiency of serotonin induced by this antimetabolite.

* With the technical assistance of G. Schaffner and E. Van Winkle.

${ }^{1}$ G. Barger, Ergot and Ergotism (London: Gurney \& Jackson, 1931); W. A. Stoll, Schweiz. Arch. Neurol. Psychiat., 68, 279, 1947.

2 D. W. Woolley and E. Shaw, Federation Proc., 12, 542, 1953.

${ }^{3}$ E. Shaw and D. W. Woolley, J. Biol. Chem., 203, 979, 1953.

4 J. H. Gaddum, C. O. Hebb, A. Silver, and A. A. B. Swan, Quart. J. Exptl. Physiol., 38, 255, 1953.

${ }^{5}$ D. W. Woolley and E. Shaw, these Proceedings, 40, 228, 1954.

B D. W. Woolley and E. Shaw, Brit. Med.J., 2, 122, 1954.

${ }^{7}$ LSD-25 was kindly supplied by Sandoz Chemical Works, Inc.

${ }^{8}$ D. W. Woolley and E. Shaw, J. Biol. Chem., 203, 69, 1953.

9 M. C. Greig and M. K. Carter, Arch. Biochem. and Biophys., 52, 175, 1954.

${ }_{10}$ M. Roche e Silva and J. Ribiero do Valle, Abstr. XIX Intern. Physiol. Congr. (1953), p. 738.

${ }^{11}$ H. A. Abramson and L. T. Evans, Science, 120, 990, 1954.

\section{FINE STRUCंTURE OF A GENETIC REGION IN BACTERIOPHAGE}

\section{By Seymour Benzer}

BIOPHYSICAL LABORATORY, DEPARTMENTS OF BIOLOGICAL SCIENYCES AND PHYSICS, PURDUE UNIVERSTTY, LAFAYETTE, INDIANA

\section{Communicated by M. Delbrück, April 6, 1955}

This paper describes a functionally related region in the genetic material of a bacteriophage that is finely subdivisible by mutation and by genetic recombination. The group of mutants resembles similar cases which have been observed in many organisms, usually designated as "pseudo-alleles." (See reviews by Lewis ${ }^{1}$ and 
Pontecorvo. ${ }^{2}$ ) Such cases are of special interest for their bearing on the structure and function of genetic determinants.

The phenomenon of genetic recombination provides a powerful tool for separating mutations and discerning their positions along a chromosome. When it comes to very closely neighboring mutations, a difficulty arises, since the closer two mutations lie to one another, the smaller is the probability that recombination between them will occur. Therefore, failure to observe recombinant types among a finite number of progeny ordinarily does not justify the conclusion that the two mutations are inseparable but can only place an upper limit on the linkage distance between them. A high degree of resolution requires the examination of very many progeny. This can best be achieved if there is available a selective feature for the detection of small proportions of recombinants.

Such a feature is offered by the case of the rII mutants of T4 bacteriophage described in this paper. The wild-type phage produces plaques on either of two bacterial hosts, $\mathrm{B}$ or $\mathrm{K}$, while a mutant of the rII group produces plaques only on B. Therefore, if a cross is made between two different rII mutants, any wild-type recombinants which arise, even in proportions as low as $10^{-8}$, can be detected by plating on $\mathrm{K}$.

This great sensitivity prompts the question of how closely the attainable resolution approaches the molecular limits of the genetic material. From the experiments of Hershey and Chase, ${ }^{3}$ it appears practically certain that the genetic information of phage is carried in its DNA. The amount of DNA in a particle of phage T2 has been determined by Hershey, Dixon, and Chase ${ }^{4}$ to be $4 \times 10^{5}$ nucleotides. The amount for $\mathrm{T} 4$ is similar. ${ }^{5}$ If we accept the model of DNA structure proposed by Watson and Crick, ${ }^{6}$ consisting of two paired nucleotide chains, this corresponds to a total length of DNA per T4 particle of $2 \times 10^{5}$ nucleotide pairs. We wish to translate linkage distances, as derived from genetic recombination experiments, into molecular units. This cannot be done very precisely at present. It is not known whether all the DNA in a phage particle is indispensable genetic material. Nor is it known whether a phage "chromosome" (i.e., the physical counterpart of a linkage group identified by genetic means) is composed of a single (duplex) DNA fiber or whether genetic recombination is equally probable in all chromosomal regions. For the purpose of a rough calculation, however, these notions will be assumed to be true. Thus we place the total linkage map of T4 in correspondence with $2 \times 10^{5}$ nucleotide pairs of DNA. The total known length of the three linkage groups ${ }^{7}$ in phage T4 amounts to some 100 units (one unit $=1$ per cent recombination in a standard cross). In addition, there is evidence ${ }^{8}$ for roughly another 100 units of length connecting two of the groups. Therefore, if we assume 200 recombination units to correspond to $2 \times 10^{5}$ nucleotide pairs, the recombination per nucleotide pair is $10^{-3}$ per cent. That is to say, given two phage mutants whose mutations are localized in their chromosomes at sites only one nucleotide pair apart, a cross between these mutants should give rise to a progeny population in which one particle in $10^{5}$ results from recombination between the mutations (provided, of course, that recombination is possible between adjacent nucleotide pairs). This computation is an exceedingly rough one and is only intended to indicate the order of magnitude of the scale factor. Some preliminary results are here presented of a program designed to extend genetic studies to the molecular (nucleotide) level. 
$r$ Mutants.-The wild-type phages $\mathrm{T} 2, \mathrm{~T} 4$, and $\mathrm{T} 6$ produce small plaques with rough edges when plated on strain B of Escherichia coli. From sectors of clearing in these plaques, mutants can be readily isolated which produce large, sharp-edged plaques (Hershey ${ }^{9}$ ). These mutants have been designated " $r$ " for rapid lysis; they differ from the wild type by a failure to cause "lysis inhibition" on strain $\mathrm{B}\left(\right.$ Doermann $\left.{ }^{10}\right)$. The wild type has a selective advantage over $r$ mutants when the two types grow together on B. The genetics of $r$ mutants was studied by Hershey and Rotman, ${ }^{11}$ who found three regions in the linkage map of $\mathrm{T} 2$ in which various mutations causing the $r$ phenotype were located, including one large "cluster" of mutants which were shown to be genetically distinct from one another. The genetic study of $\mathrm{T} 4$ by Doermann and Hill ${ }^{7}$ showed $\mathrm{r}$ regions corresponding to two of those in T2. T6 also has at least two such $\mathrm{r}$ regions.

The rII Group.-For all three phages, T2, T4, and T6, the r mutants can be separated into groups on the basis of their behavior on strains other than B. This paper will be concerned only with one group, which will be called the "rII group." Mutants of the rII group are distinguished from those of other groups, and from wild type, by a failure to produce plaques on certain lysogenic strains ${ }^{12}$ of $E$. coli which carry phage $\lambda$. As shown in Table 1, a mutant of the rII group produces

TABLE 1

Phenotypes (Plaque Morphology) of T4 Wild and rII Mutant Plated on Various Hosts

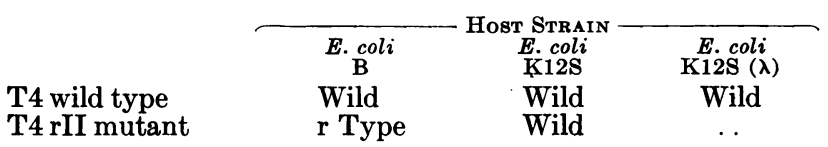

r-type plaques on strain $\mathrm{B}$, wild-type plaques on strain $\mathrm{K} 12 \mathrm{~S}$ (nonlysogenic strain sensitive to $\lambda$ ), and no plaques on K12S $(\lambda)$ (derived from K12S by lysogenization with $\lambda$ ). The wild-type phage produces similar plaques on all three strains. In the case of $\mathrm{T} 4$, with which we shall be concerned in this paper, the efficiencies of plating are approximately equal on the three strains, except, of course, for rII on K12S $(\lambda)$. The three bacterial strains will be here designated as "B," "S," and "K."

Approximately two-thirds of the independently arising $\mathrm{r}$ mutants isolated on B are of the rII type. This group includes the "cluster" of $r$ mutants of T2 described by Hershey and Rotman and the r47 and r51 mutants described by Doermann and Hill in the corresponding map region of $\mathrm{T} 4$ but does not include $\mathrm{r}$ mutants located outside that region. Similarly, all newly isolated mutants showing the rII character have turned out to fall within the same region, as indicated in Figure 1.

The properties of the rII group are especially favorable for detailed genetic study. An rII mutant has three different phenotypes on the three host strains (Table 1): (1) altered plaque morphology on B, (2) indistinguishable from wild type on S, and (3) unable to produce plaques on $\mathrm{K}$. These properties are all useful. By virtue of their altered plaque type on $\mathrm{B}, \mathrm{r}$ mutants are readily isolated, and those of the rII group are identified by testing on $\mathrm{K}$. Where it is desired to avoid a selective disadvantage compared with wild type, e.g., in measuring mutation 
rates, $\mathrm{S}$ can be used as a nondiscriminating host. The failure of rII mutants to plate on $\mathrm{K}$ enables one to detect very small proportions of wild-type particles due to reversion or due to recombination between different rII mutants.

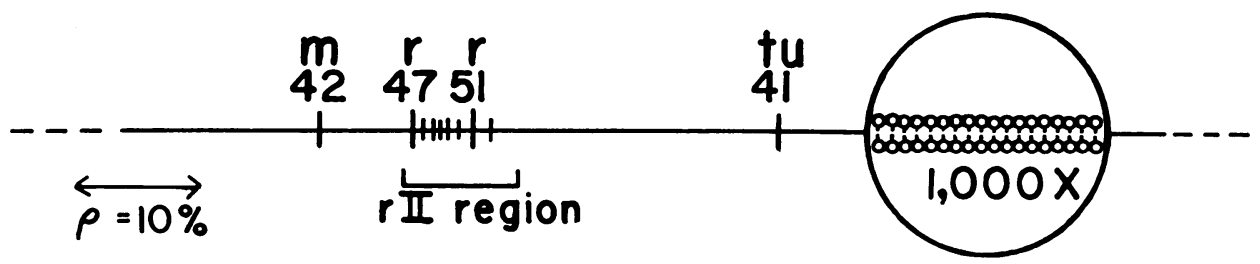

FIG. 1.-Partial linkage map of $\mathrm{T} 4$ (Doermann), indicating the location of the rII region. $m$ and $t u$ designate "minute plaque" and "turbid plaque" mutations. The circular inset shows, diagrammatically, the corresponding dimensions of the IDNA chain magnified 1,000 diameters.

Fate of rII Mutants in K.-Wild-type and rII mutants adsorb equally well to strains $\mathbf{S}$ and $\mathrm{K}$. Whereas the wild type provokes lysis and liberation of a burst of progeny on both strains, the rII mutant grows normally only on S. Infection of $\mathrm{K}$ with an rII mutant provokes very little (and/or very late) lysis, although all infected cells are killed. The block in growth of rII mutant is associated with the presence of the carried phage $\lambda$. The reason for this association is unknown.

Quantitative Differences in Phenotype.-While all rII mutants show the same phenotypic effect of poor multiplication on $K$, they differ in the degree of this effect. A certain proportion of $\mathrm{K}$ infected with rII actually liberates some progeny, which can be detected by plating the infected cells on $B$. The fraction of infected cells yielding progeny defines a "transmission coefficient" characteristic of the mutant. The transmission coefficient is insensitive to the multiplicity of infection but depends strongly upon the physiological state of the bacteria $(\mathbf{K})$ and upon temperature. Under given conditions, however, the coefficient can be used as a comparative index of degree of phenotypic effect, a "leaky" mutant having a high coefficient. As can be seen in Table 2, a wide range of values is found.

TABLE 2

\begin{tabular}{lccc}
\multicolumn{4}{c}{ Properties of T4 } \\
$\begin{array}{c}\text { Mutant } \\
\text { Number }\end{array}$ & $\begin{array}{c}\text { Map } \\
\text { Position }\end{array}$ & $\begin{array}{c}\text { Transmission } \\
\text { Coefficient }\end{array}$ & $\begin{array}{c}\text { Reversion } \\
\text { Index } \\
\text { (units of 10-6) }\end{array}$ \\
r47 & 0 & 0.03 & $<0.01$ \\
r104 & 1.3 & .91 & $<1$ \\
r101 & 2.3 & .03 & 4.5 \\
r103 & 2.9 & .02 & $<0.2$ \\
r105 & 3.4 & .02 & 1.8 \\
r106 & 4.9 & .55 & $<1$ \\
r51 & 6.7 & .02 & 170 \\
r102 & 8.3 & .02 & $<0.01$
\end{tabular}

\footnotetext{
* Three parameters are given for each mutant. The map position is computed from the sum of the nearest intervals shown in Figure 2 and is given in percentage recombination units, taking the position of $\mathrm{r} 47$ as zero. The "transmission coefficient" is a measure of phenotypic effect determined by infecting bacteria $K$ with the mutant in question and is given as the fraction of such infected cells yielding plaques on strain B. The "reversion index" in question and is given as the fraction of such infected cells yielding plaques on strain B. The "reversion index"
is the average fraction of wild-type particles arising in lysates of the mutant grown from a small inoculum on a nonis the average
selective host.
}

Plaques on $\mathrm{K}$.- Some rII mutants produce no plaques on $\mathrm{K}$, even when as many as $10^{8}$ particles (as measured by plaque count on B) of a stock are plated. Other rII mutants, however, produce various proportions of plaques on $K$. When the 
plaques appearing on $\mathrm{K}$ are picked and retested, they fall into three categories: (1) a type which, like the original mutant, produces very few plaques on $\mathrm{K}$ and $\mathrm{r}$ type plaques on B; (2) a type which produces plaques (often smaller than wild type) on $\mathrm{K}$ with good efficiency but r-type plaques on $\mathrm{B}$; and (3) a type indistinguishable from the original wild. These three types are understood to be due to the following: (1) "leaking" effects, i.e., ability of the mutant to grow slightly on $\mathrm{K}$, so that there is a chance for a few visible plaques to form; (2) a mutation which partially undoes the effect of the rII mutation, so that multiplication in $K$ is possible, but the full wild phenotype is not achieved; and (3) apparent reverse mutation, which may or may not be genuine, to the original wild type.

The proportion of each type occurring in a stock is characteristic and reproducible for a particular rII mutant but differs enormously from one rII mutant to another. There is no evident correlation in the rates of occurrence of the three types.

Reversion Rates of rII Mutants.-Reversion of $\mathrm{r}$ mutants to a form indistinguishable from wild type was demonstrated by Hershey, ${ }^{9}$ who made use of the selective advantage of wild type on $B$ to enrich its proportion in serial transfers. Given the inability of rII mutants to produce plaques on $\mathrm{K}$, such reversions are easily detected, even in very small proportion. An index to the frequency of reversion of a particular rII mutant can be obtained by preparing a lysate from a small inoculum (about 100 particles, say, so that there is very little chance of introducing a wildtype particle present in the stock). If $\mathrm{S}$ is used as the host, both rII mutant and any reversions which arise can multiply with little selection, as shown by control mixtures. The average fraction of wild-type particles present in several lysates is an index which can be shown to be roughly proportional to the probability of reversion per duplication of the rII mutant. Under the conditions of measurement the index is of the order of 10-20 times the probability of reversion per duplication. The plaques appearing on $\mathrm{K}$ must be tested by picking and replating on $\mathrm{B}$. This eliminates the "spurious" plaques produced by partial reversions and by leaky mutants, which show up as $\mathrm{r}$ type on $\mathrm{B}$. As may be seen in Table 2, the reversion indices for rII mutants vary over a very wide range. One mutant has been found which reverts 10 times more frequently than $\mathrm{r} 51$, so that the reversion rates cover a known range of over $10^{5}$-fold.

It has not been proved that these apparent reversions constitute a genuine return to the original wild type. However, the possibility of suppressor mutations distant from the site of the rII mutation has been ruled out by backcrosses to the original wild type. Krieg ${ }^{13}$ found very few, if any, r-type recombinants in backcrosses of several reversions, localizing the reverse changes to within a few tenths of a per cent linkage distance from the original rII mutations. One case of "partial reversion" has also been tested by backcrossing, and failure to observe rIItype recombinants localized the "partial reverse mutation" to within the rII region.

Mapping of the rII Region.-A cross between two rII mutants is made by infecting a culture of $\mathrm{B}$ with equal multiplicities (three per bacterium) of each type. The yield after lysis contains the two parental types and, if the parents are genetically distinct, two recombinant types, the double mutant and wild type. In the average yield from many cells, the recombinant types occur in equal numbers. ${ }^{11}$ In all cases thus far tested, double rII mutants, like single mutants, do not produce plaques on $K$. On the assumption that this is generally true, the proportion of 
recombinants in the yield can be measured simply by doubling the ratio of the plaque count on $K$ (which registers only the wild recombinant) to the count on $B$ (which registers all types). The percentage of wild type thus measured agrees well with a direct count of plaque types on $B$.

In this way, a series of six rII mutants of T4 (the first six isolated-not selected in any way) have been crossed with each other and with r47 and r51 (kindly supplied by A. H. Doermann) in 23 of the 28 possible pairs. The results of these crosses are given in Figure 2 and are compatible with the indicated seriation of the mutants. The distances are only roughly additive; there is some systematic deviation in the sense that a long distance tends to be smaller than the sum of its component shorter ones. Part of this discrepancy is accounted for by the Visconti-Delbrück correction for multiple rounds of mating. ${ }^{14}$ Reversion rates were small enough to be negligible in these crosses. Thus, while all rII mutants in this set fall into a small portion of the phage linkage map, it is possible to seriate them unambiguously, and their positions within the region are well scattered.

Tests for Pseudo-allelism.-The functional relatedness of two closely linked mutations causing similar defects may be tested by constructing diploid heterozygotes containing the two mutations in different configurations. ${ }^{1,2}$ The $c i s$ form, with both mutations in one chromosome, usually behaves as wild type, since the second chromosome supplies an intact functional unit (or units). However, the trans form, containing one of the mutations in each chromosome, may or may not produce the wild phenotype. If it does, it is

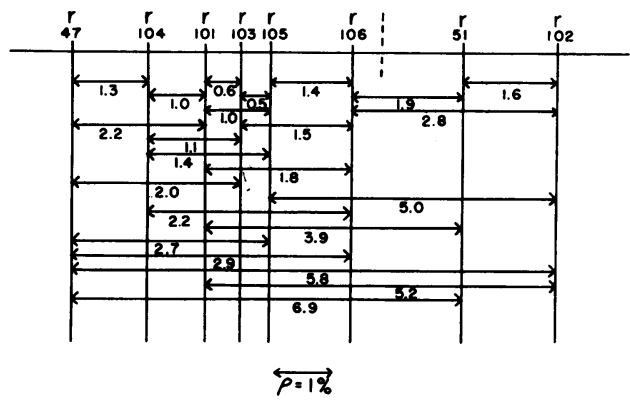

FIg. 2.-Larger-scale map of eight rII mutants, including Doermann's r47 and r51. Newly isolated mutants are numbered starting with 101. The recombination value (in per cent) for each cross is obtained by plating the progeny on $K$ and on $B$ and doubling the ratio of plaque count on $K$ to count on $B$. concluded that the two mutations in question are located in separate functional units.

In applying this test to the rII mutants, the diploid heterozygote can be simulated by a mixed infection with two kinds of phage. The rII phenotype is a failure to lyse $\mathrm{K}$, whereas the wild phenotype is to cause lysis. If $\mathrm{K}$ is mixedly infected with wild type and rII mutant, the cells lyse, liberating both types of phage. Thus the presence of wild type in the cell supplies the function which is defective in rII type, and the rII mutation can be considered "recessive." Although it has not yet been tested, the cis configuration of double rII mutant plus wild type is also presumed to produce lysis in all cases. The trans configuration is obtained by infecting $\mathrm{K}$ with the pair of rII mutants in question. This is found to give lysis or not, dedepending upon which rII mutants compose the pair. The results are summarized by the dotted line in Figure 2, indicating a division of the rII region into two segments. If both mutants belong to the same segment, mixed infection of $\mathrm{K}$ gives the mutant phenotype (very few cells lyse). If the two mutants belong to different segments, extensive lysis occurs with liberation of both infecting types (and recombinants). These results are summarized in Figure 3. Thus, on the basis of this 
test, the two segments of the rII region correspond to independent functional units.

Actually, for mixed infection of $\mathrm{K}$ with two (nonleaky) mutants of the same segment, a very small proportion of the cells do lyse and liberate wild recombinants, that proportion increasing with the linkage distance between the mutations. For two rII mutants separated by 1 per cent linkage distance (measured by a standard cross on $\mathrm{B}$ ) the proportion of mixedly infected $\mathrm{K}$ yielding any wild particles is about 0.2 per cent.

This value has bearing upon the effect upon $\mathrm{K} / \mathrm{B}$ values of the heterozygous phage particles which arise in a cross between two rII mutants on B. In such a cross between closely linked rII mutants, the progeny should include about 2 per cent of particles containing a trans configuration heterozygous piece. ${ }^{15}$ When one of these is plated on $\mathrm{K}$, there is a certain chance that a wild recombinant may form in the first cycle of infection, leading to production of a plaque. If it is assumed that these are no more likely to do so than a mixed infection of $\mathrm{K}$ with two complete mutant particles, it can be concluded that the effect of these heterozygous particles upon the count on $\mathrm{K}$ is negligible, provided that both $\mathrm{rII}$ mutants belong to the same segment. For mutants in different segments, however, the "effi-

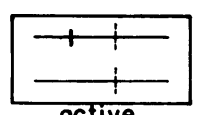

octive

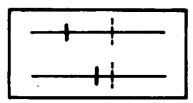

inoctive
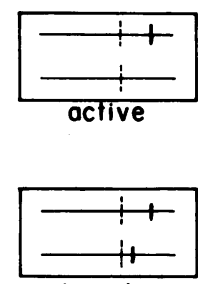

inoctive
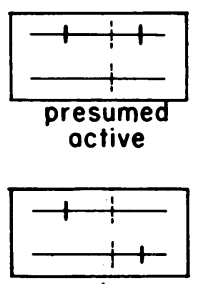

active

Fig. 3.-Summary of tests for "position-effect pseudo-allelism" of rII mutants. Each diagram represents a diploid heterozygote as simulated by mixed infection of a bacterium $(K)$ with two types of phage containing the indicated mutations. Active means extensive lysis of the mixedly infected cells; inactive means very little lysis. The dotted line represents a dividing point in the rII region, the position of which is defined by these results. ciency" of the heterozygous particles should be much greater, and recombination values measured by the $\mathrm{K} / \mathrm{B}$ method should run considerably higher than the true values. The recombination values in Figure 2 for crosses which transgress the segmental divide are probably subject to some correction for this reason.

Rough Mapping by Spot Test.-If a stock of either of two rII mutants is plated on $\mathrm{K}$, no plaques arise; but if both are plated together, some bacteria become infected by both mutants and, if this leads to the occurrence of wildtype recombinants, plaques are produced. If the two mutants are such that wild recombinants cannot arise between them (e.g., if they contain identical mutations), no plaques appear. A given rII mutant may thus be tested against several others on a single plate by first seeding the plate with $\mathrm{K}$ plus the mutant in question (in the usual soft agar top layer) and then spotting with drops containing the other rII mutants.

Inspection of such a plate immediately places the unknown mutant in the proper segment, since spotting any mutant of segment A against any mutant of segment $B$ gives a very clear spot, due to the extensive lysis of mixedly infected bacteria. However, for a pair of mutations belonging to the same segment, plaques are produced only by the relatively few mixedly infected bacteria which give rise to wild recombinants. The greater the linkage distance between the mutations, the larger the number of plaques that appear in the spot. A group of mutants of the same segment may thus be seriated by seeding one plate with each and spotting with all the others. Given a previously seriated group, a new mutant can thus be quickly 
located within the group. This method works best for mutants which are stable (i.e., low reversion rate) and nonleaky, so that large numbers of phage particles can be plated. Reversions or pronounced leaking effects obviously cause an obscuring background.

This test has been applied to a large group of stable, nonleaky rII mutants. Their approximate locations as deduced from these tests are shown in Figure 4. Some of the mutants showed anomalies which made it impossible to locate them as members of a series. They gave very little recombination with any of the mutants located within a certain span, while behaving normally with respect to mutants located outside that span. They are indicated in Figure 4 by horizontal lines extending over the span.

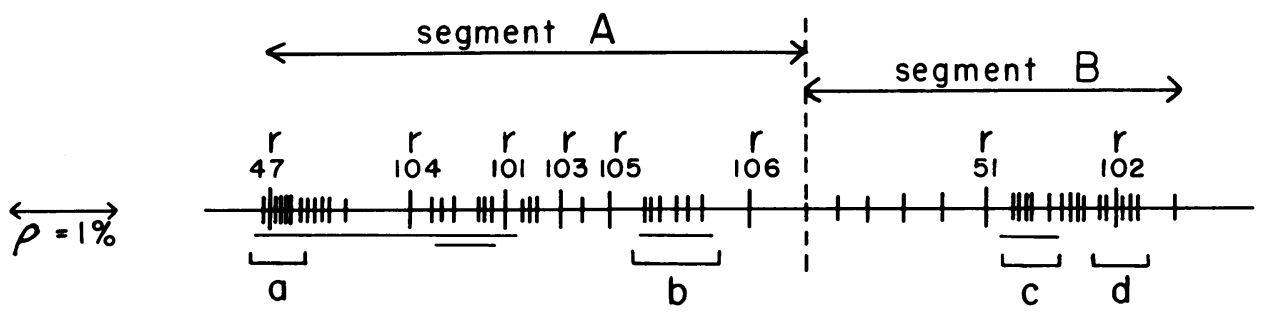

FIG. 4.-Preliminary locations of various rII mutants, based upon spot tests

Spot tests on numerous other mutants have shown that mutants of varied reversion rates, transmission coefficients, and rates of "partial reversion" occur at scattered positions in both segments.

Mapping of "Microclusters." - The spot test enables us to pick out "microclusters," i.e., groups of very closely neighboring mutations. Four such groups selected for further study are indicated in Figure 4, and the results of mapping them are given in Figure 5. While some intervals show reasonably good additivity properties, there are some mutants which give violently anomalous results. Thus in microcluster $a, \mathrm{r} 47$ gives no wild recombinants (i.e., less than 1 in $10^{6}$ ) with any of the other three mutants, but two pairs of the three do show recombination. These results can be understood if it is assumed that each mutation extends over a certain length of the chromosome, and production of wild type requires recombination within the space between those lengths. According to this interpretation, the mutations would cover the lengths indicated by the bars in Figure 5. These anomalies resemble those observed in the spot tests, only they are more limited in span.

This observation raises the question of whether there exist true "point" mutations (i.e., involving an alteration of only one nucleotide pair) or whether all mutations involve more or less long pieces of the chromosome. It must be remembered that the mutants used in these experiments were selected for extreme stability against reversion. This procedure would be expected to enrich the proportion of mutants containing gross chromosomal alterations. So far as is known, the anomalous cases observed could equally well be imagined to be due to double (i.e., two near-by "point") mutations, inversions, or deletions of the wild-type chromosome. In continuing these experiments, it would seem well advised to employ only mutants for which some reversion is observed. 

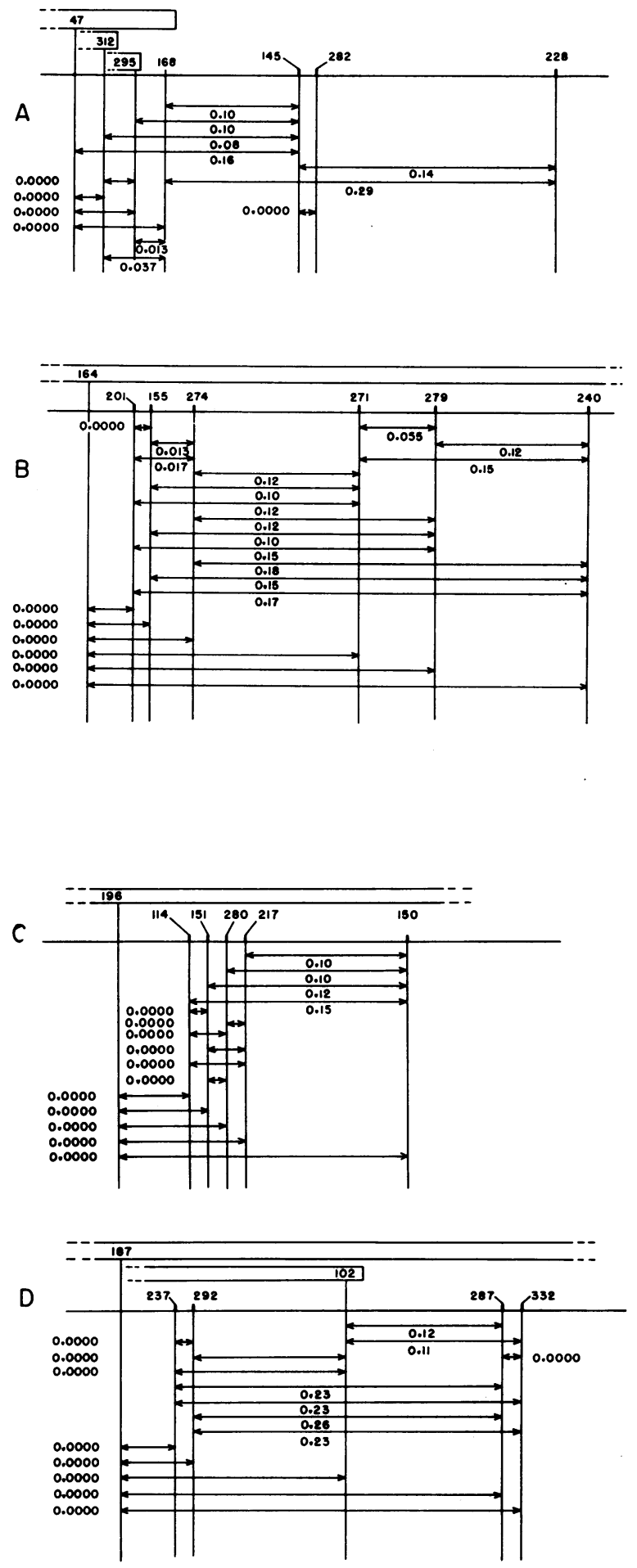

Fig. 5.-Maps of microclusters 
Discussion.-The set of rII mutants defines a bounded region of a linkage group in which mutations may occur at various locations, all the mutations leading to qualitatively similar phenotypic effects. The rII region would seem, therefore, to be functionally connected, so that mutations arising anywhere within the region affect the same phenotype. This effect is expressed, in case strain B is the host, by failure to produce lysis inhibition; in case $S$ is the host, by no consequence; and in case $\mathrm{K}$ is the host, by inability to multiply normally. The failure of an rII mutant to mature in $\mathrm{K}$ can be overcome by the presence of a wild-type phage in the same cell. This could be understood if the function of the region in the wild-type "chromosome" were to control the production of a substance or substances needed for reproduction of this phage in $\mathrm{K}$ cells.

The phenotypic test for "pseudo-allelism" leads to the division of the region into two functionally distinguishable segments. These could be imagined to affect two necessary sequential events or could go to make up a single substance the two parts of which must be unblemished in order for the substance to be fully active. For example, each segment might control the production of a specific polypeptide chain, the two chains later being combined to form an enzyme. While it is not known whether this sort of picture is applicable, a model of this kind is capable of describing the observed properties of the rII mutants. The map position of a mutation would localize a change in the region (and also in the "enzyme" molecule), the reversion rate would characterize the type of change involved in the genetic material, and the degree of phenotypic effect would be an expression of the degree of resultant change in the activity of the enzyme. A "leaky" mutant would be one where this latter effect was small. While no obvious correlation has yet been observed among these three parameters of rII mutants, one may well show up upon more exhaustive study.

"Clustering" of similar mutants separable by crossing-over has been observed for several characters in phage by Doermann and Hill and appears to represent the rule. This may well be the rule in all organisms, simply because functional genetic units are composed of smaller recombinational and mutational elements. One would expect to see this effect more readily in phage because the probability of recombination per unit of hereditary material is much greater than for higher organisms.

By extension of these experiments to still more closely linked mutations, one may hope to characterize, in molecular terms, the sizes of the ultimate units of genetic recombination, mutation, and "function." Our preliminary results suggest that the chromosomal elements separable by recombination are not larger than the order of a dozen nucleotide pairs (as calculated from the smallest non-zero recombination value) and that mutations involve variable lengths which may extend over hundreds of nucleotide pairs.

In order to characterize a unit of "function," it is necessary to define what function is meant. The entire rII. region is unitary in the sense that mutations anywhere within the region cause the rII phenotype. On the basis of phenotype tests of trans configuration heterozygotes, this region can be subdivided into two functionally separable segments, each of which is estimated to contain of the order of $4 \times 10^{3}$ nucleotide pairs. If one assumes that each segment has the "function" of specifying the sequence of amino acids in a polypeptide chain, then the specification of each individual amino acid can as well be considered a unitary function. It 
would seem feasible, with this system, to extend genetic studies even to the level of the latter functional elements.

Summary.-It has been discovered that the mutations in the rII region of phage T4 have a characteristic in common which sets them apart from the mutations in all other parts of the map. This characteristic is a host-range reduction, namely, a failure to produce plaques on a host $(K)$ lysogenic for phage $\lambda$. The mutant phage particles adsorb to and kill $\mathrm{K}$, but normal lysis and phage release do not occur.

All mutants with this property are located within a sharply defined portion of the phage linkage map. Within that region, however, their locations are widely scattered. An unambiguous seriation of the mutants, with roughly additive distances, can be accomplished, excepe for certain anomalous cases.

The simultaneous presence of a wild-type phage particle in $\mathrm{K}$ enables the multiplication of rII mutants to proceed, apparently by supplying a function in which the mutant is deficient. A heterozygous diploid in the trans configuration is simulated by a mixed infection of $\mathrm{K}$ with two mutant types. The application of the phenotype test to pairs of rII mutants leads to the division of the region into two functionally separable segments.

Spontaneous reversion to wild-type had been observed for most of these mutants. It remains to be seen whether these are genuine reversions. Each mutant reverts at a characteristic rate, but the rates for different mutants differ enormously. Partial reversions to intermediate types are also observed.

The mutants differ greatly in degree of residual ability to grow on $\mathrm{K}$. There is no evident correlation between map position, reversion rate, and degree of residual activity of the various mutants.

The selective feature of $\mathrm{K}$ for wild-type recombinants offers the possibility of extending the recombination studies to an analysis of the fine details of the region.

Preliminary studies of this type indicate that the units of recombination are not larger than the order of one dozen nucleotide pairs and that mutations may involve various lengths of "chromosome."

I am much indebted to A. D. Hershey and A. H. Doermann for stocks of their genetically mapped mutants, to Sydney Brenner and David Krieg for stimulating discussion, and to Max Delbrück for his invaluable moderating influence.

* Supported by a grant-in-aid from the American Cancer Society upon recommendation of the Committee on Growth of the National Research Council.

1 E. B. Lewis, Cold Spring Harbor Symposia Quant. Biol., 16, 159-174, 1951.

2 G. Pontecorvo, Advances in Enzymol., 13, 121-149, 1952.

3 A. D. Hershey and M. Chase, J. Gen. Physiol., 36, 39-56, 1952.

4 A. D. Hershey, J. Dixon, and M. Chase, J. Gen. Physiol., 36, 777-789, 1953.

5 E. K. Volkin, personal communication.

6 J. D. Watson and F. H. C. Crick, Cold Spring Harbor Symposia Quant. Biol., 18, 123-131, 1953.

7 A. H. Doermann and M. B. Hill, Genetics, 38, 79-90, 1953.

$8 \mathrm{G}$. Streisinger and V. Bruce, personal communication.

9 A. D. Hershey, Genetics, 31, 620-640, 1946.

10 A. H. Doermann, J. Bacteriol., 55, 257-276, 1948.

11 A. D. Hershey and R. Rotman, Genetics, 34, 44-71, 1949.

12 E. M. Lederberg and J. Lederberg, Genetics, 38, 51-64, 1953.

${ }_{13} \mathrm{D}$. Krieg, personal communication.

14 N. Visconti and M. Delbrück, Genetics, 38, 5-33, 1953.

${ }^{15}$ A. D. Hershey and M. Chase, Cold Spring Harbor Symposia Quant. Biol., 16, 471-479, 1951;

C. Levinthal, Genetics, 39, 169-184, 1954. 